\title{
THE CYCLIC HOMOLOGY AND $K$-THEORY OF CURVES
}

\author{
S. GELLER, L. REID AND C. WEIBEL
}

\begin{abstract}
It is now possible to calculate the $K$-theory of a large class of singular curves over fields of characteristic zero. Roughly speaking, the $K$-theory of a curve is the $K$-theory of its (smooth) normalization plus a few shifted copies of the $K$-theory of the field plus a "nil part." The nil part is a vector space depending only on the analytic type of the singularities, and may be computed locally. We completely compute the nil part for seminormal curves and give a conjectural calculation in general which depends upon cyclic homology.
\end{abstract}

Until recently, very little has been known about the higher algebraic $K$ theory of anything but finite fields. In this note we announce the computability of the $K$-theory of singular curves in characteristic zero in terms of the $K$-theory of smooth curves and fields. If the curve is seminormal, we give a complete calculation; otherwise, the calculation depends on the validity of:

CONJECTURE. Let $B$ be a finite integral extension of a ring $A$, and let $I$ be the conductor ideal. Assume $A$ contains $\mathbf{Q}$, the rational numbers. Then the map

$$
K_{*}(A, B, I) \rightarrow H C_{*-1}(A, B, I)
$$

is an isomorphism, where the right-hand term is double relative cyclic homology taken over the field $\mathbf{Q}$.

This conjecture is known when $B=A / J[\mathbf{O W}]$. In the absence of this conjecture, all our results can be interpreted as calculations of the cyclic homology of affine curves. In order to more simply present our results, let us set

$$
V_{n}= \begin{cases}0 & n=0,1, \\ k \oplus \Omega_{k}^{2} \oplus \Omega_{k}^{4} \oplus \cdots \oplus \Omega_{k}^{n-2} & n \text { even, } n \geq 2, \\ \Omega_{k} \oplus \Omega_{k}^{3} \oplus \cdots \oplus \Omega_{k}^{n-2} & n \text { odd, } n \geq 3 .\end{cases}
$$

Here $\Omega_{k}^{i}$ denotes the $i$ th exterior of the module $\Omega_{k}$ of Kähler differentials of $k$ over $\mathbf{Q}$. As an illustration, we present

CURVE 1 (TWO INTERSECTING LINES). Let $k$ be a field of characteristic zero, and set $A=k[x, y] /(x y), I=(x, y) A$, and $X=\operatorname{Proj}(k[X, Y, Z] /(X Y))$.

Received by the editors June 12, 1986.

1980 Mathematics Subject Classification (1985 Revision). Primary 14F15, 18F25; Secondary 19E08, 19D25.

Key words and phrases. Algebraic $K$-theory, cyclic homology, singular curve.

Research partially supported by National Science Foundation Grant DMS-8501135 (S. Geller), and National Science Foundation Grant DMS-8503018-01 (C. Weibel). 
Then

$$
\begin{aligned}
H C_{n}(A) & =H C_{n}(k) \oplus V_{n+1} \oplus\left(I \otimes \Omega_{k}^{n}\right), \\
K_{n}(A) & =K_{n}(k) \oplus V_{n}, \\
K_{n}(X) & =K_{n}(A) \oplus K_{n}(k) \oplus K_{n}(k) .
\end{aligned}
$$

Fix a field $k$ of characteristic zero and let $X$ be a singular curve over $k$. We shall compute the $K$-theory of $X$ by means of a series of reductions. Using analytic isomorphisms [W1], we reduce to the case in which $X$ is affine, say $X=\operatorname{Spec}(A)$. If $A$ is not reduced, we can compute the $K$-theory of $A$ in terms of the $K$-theory of $A_{\text {red }}=A / N(N$ is the nilradical of $A)$ and the relative groups of $N$. The latter are computable in terms of cyclic homology; namely, $K_{n}(A, N) \cong H C_{n-1}(A, N)$. (This isomorphism is due to Goodwillie $[\mathbf{G}]$.) We thus reduce to the case in which $A$ is reduced.

At this point, we break up the $K$-theory of $A$ into two pieces: the KaroubiVillamayor theory and the nil $K$-theory [W2]. The groups $K V_{*}(A)$ are easily computed in terms of the $K$-theory of the normalization $B$ of $A$ and the residue fields of $A$ and $B$ at the singular points of $A$. This procedure is outlined in [R and W3].

The groups nil $K_{*}(A)$ are $Q$-vector spaces, and it is these that we can compute. Since $A$ is reduced and 1-dimensional, nil $K_{*}(A)$ is the direct sum over the singular primes $m$ of $A$ of the groups nil $K_{*}\left(A_{m}\right)$. These groups depend only on the analytic type of the singularity, in the sense that if $\hat{A}$ is the $m$-adic completion of $A$, then nil $K_{*}\left(A_{m}\right) \cong \operatorname{nil} K_{*}(\hat{A})$.

To illustrate the nature of the last few reductions, the computation for two intersecting lines allows us to compute

Curve 2 (Node). Let $A=k[x, y] /\left(y^{2}=x^{2}-x^{3}\right)$ and

$$
X=\operatorname{Proj}\left(k[X, Y, Z] /\left(Y^{2} Z=X^{2} Z-X^{3}\right)\right) .
$$

Then $K_{n}(X)=K_{n}(A) \oplus K_{n}(k)$ and

$$
K_{n}(A)=K_{n}(k) \oplus K_{n+1}(k) \oplus V_{n} .
$$

Similarly, we can calculate the $K$-theory of any curve whose singularities have linearly independent branches by means of the above reductions and the following special case.

Curve $3(b+1$ BRAnChes $)$. Let $A=k\left[x_{0}, \ldots, x_{b}\right] /\left(x_{i} x_{j}=0, i \neq j\right)$. Then

$$
\begin{aligned}
H C_{n}(A) & =H C_{n}(k) \oplus \coprod_{c(b, n+1)} k \oplus \coprod_{c(b, n)} \Omega_{k} \oplus \cdots \oplus \coprod_{c(b, 2)} \Omega_{k}^{n-1} \oplus\left(I \otimes \Omega_{k}^{n}\right), \\
K_{n}(A) & =K_{n}(k) \oplus \coprod_{c(b, n)} k \oplus \prod_{c(b, n-1)} \Omega_{k} \oplus \cdots \oplus \coprod_{c(b, 2)} \Omega_{k}^{n-2},
\end{aligned}
$$

where, letting $\mu$ denote the Möbius function,

$$
c(b, q)=\sum_{\substack{e \mid q \\ q-e \text { even }}} \sum_{d \mid e} \frac{\mu(e / d)}{e}\left[b^{d}+(-1)^{d} b\right] .
$$


The function $c(b, q)$ counts the number of ways of using the alphabet $\{0,1, \ldots, b\}$ to write words of $q$ letters around a circle so that (i) no two adjacent letters are the same and (ii) if rotation by $e$ positions fixes the word then $(-1)^{e}=(-1)^{q}$. (For example, the word 012012 is disallowed when $q=6$.) Since

$c(1, q)$ is 1 if $q$ is even and 0 if $q$ is odd, the case $b=1$ recovers Curve 1 . Note that $c(b, 2)=\left(b^{2}+b\right) / 2, c(b, 3)=\left(b^{3}-b\right) / 3, c(b, 4)=\left(b^{4}-b^{2}\right) / 4+\left(b^{2}+b\right) / 2$. In general $c(b, q) \sim b^{q} / q$.

The elements of $K_{n}(A)$ are easy to describe using "Loday symbols" $\left\langle\left\langle f_{1}, \ldots, f_{q}\right\rangle\right\rangle[\mathbf{L}]$. For each of the $c(b, q)$ words $i_{1}, \ldots, i_{q}$ and each element $a_{0} d a_{1} \wedge \cdots \wedge d a_{p}$ of $\Omega_{k}^{p}$ we get an element

$$
\left\langle\left\langle a_{0} x_{i_{1}}, \ldots, x_{i_{q}}\right\rangle\right\rangle \cup\left\{a_{1}, \ldots, a_{p}\right\} \in K_{p+q}(A) .
$$

Finally, according to $[\mathbf{D}]$, every seminormal singularity has the analytic type of $A^{G}$, where $A$ is the ring of Curve 3 and $G$ is a galois group acting on both $k$ and $A$ in a graded way. Since the galois group acts on the $H C$ and $K$-groups of $A$ in an obvious way, we have

$$
\begin{gathered}
H C_{n}\left(A^{G}\right)=H C_{n}\left(k^{G}\right) \oplus\left(\coprod_{c(b, n+1)} k\right)^{G} \oplus \cdots \oplus\left(I \otimes \Omega_{k}^{n}\right)^{G}, \\
K_{n}\left(A^{G}\right)=K_{n}\left(k^{G}\right) \oplus\left(\coprod_{c(b, n)} k\right)^{G} \oplus \cdots \oplus\left(\coprod_{c(b, 2)} \Omega_{k}^{n-2}\right)^{G} .
\end{gathered}
$$

This completes the computation of the $K$-theory of seminormal curves.

Our results for nonordinary singularities are much less complete, if for no other reason than our lack of a reasonable classification. We mention just one: Then

CURVE 4 (CUSP). Let $A=k[x, y] /\left(x^{3}=y^{2}\right)=k\left[t^{2}, t^{3}\right]$, and $I=(x, y) A$.

$$
H C_{n}(A)=H C_{n}(k) \oplus V_{n+1} \oplus V_{n+1} \oplus\left(I \otimes \Omega_{k}^{n}\right)
$$

and $K_{n}(A)$ maps onto $K_{n}(k) \oplus V_{n} \oplus V_{n} \oplus \Omega_{k}^{n}$ for $n \leq 3$. Moreover, if the Conjecture mentioned above is correct, then for all $n$,

$$
K_{n}(A)=K_{n}(k) \oplus V_{n} \oplus V_{n} \oplus \Omega_{k}^{n} .
$$

Note that the groups $K_{n}(A)$ for $n=0,1$ are known to agree with the above formula $[\mathbf{K r}, 12.1]$.

\section{REFERENCES}

[D] E. Davis, On the geometric interpretation of seminormality, Proc. Amer. Math. Soc. 68 (1978), 1-5.

[G] T. Goodwillie, Algebraic K-theory and cyclic homology, preprint, 1985.

[L] J.-L. Loday, Symboles in K-théorie algébrique supérieure, C.R. Acad. Sci. Paris 292 (1981), 863-866.

$[\mathbf{K r}] \mathrm{M}$. Krusemeyer, Fundamental groups, algebraic $K$-theory and a problem of $A b$ hyankar, Invent. Math. 19 (1973), 15-47.

[OW] C. Ogle and C. Weibel, Relative algebraic K-theory and cyclic homology (in preparation). 
$[\mathbf{R}]$ L. Roberts, The $K$-theory of some reducible affine curves: a combinatorial ap proach, Lecture Notes in Math., vol. 551, Springer-Verlag, 1976.

[W1] C. Weibel, K-theory and analytic isomorphisms, Invent. Math. 61 (1980), 177197.

[W2] , Nil K-theory maps to cyclic homology, preprint, 1986.

[W3] _ , Nilpotence in K-theory, J. Algebra 61 (1979), 298-307.

Department of Mathematics, Texas A \& M University, College StaTION, TEXAS 77843

Department of Mathematics, University of Utah, Salt lake City, Utah 84112

Department of Mathematics, Rutgers University, New Brunswick, NEW JERSEY 08903 